\title{
Serum immune complexes containing IgA appear to predict erosive arthritis in a longitudinal study in rheumatoid arthritis*
}

\author{
MARIE-LOUISE WESTEDT, ${ }^{1}$ MOHAMED R DAHA, ${ }^{2}$ WILLIAM M \\ BALDWIN III, ${ }^{2}$ THEO STIJNEN, ${ }^{3}$ AND ARNOLD CATS
}

From the Departments of ${ }^{1}$ Rheumatology and ${ }^{2}$ Nephrology, University Hospital, Leiden; and the ${ }^{3}$ Department of Medical Statistics, University of Leiden, The Netherlands

SUMMARY Fifty seven patients with rheumatoid arthritis (RA) were studied longitudinally, and the presence of rheumatoid factor (RF) and various types of immune complexes (IC) was correlated with joint activity and the presence of extra-articular features (EAF). In a cross sectional study it was found that the levels of circulating IC and RF correlated significantly with joint disease activity and the presence of EAF. Longitudinally, levels of IC measured by the Clq binding activity and IC containing IgG and IgM correlated significantly with fluctuations in joint disease activity, whereas IC containing IgG and IgA correlated with the occurrence of EAF. RF and IC levels, however, did not predict the clinical course of the disease. IC containing C3 and C4 were found infrequently and were only present in patients with active rheumatoid vasculitis (RV). The continuous presence of these IC appeared to be linked to the recurrence of vasculitis, irrespective of treatment. Significantly more erosions of hands and feet were found after one year follow up in those RA patients who presented early (disease duration less than one year) who initially had a raised serum IgA IC level $(r=0.72 ; p<0.005)$.

Key words: prognostic value, articular activity, extra-articular disease, erosions.

During the last decade many reports have been published on numerous immune complex assays used to investigate the serum or synovial fluid (SF), or both, in autoimmune disease such as rheumatoid arthritis (RA). The presence of immune complexes (IC) has been implicated in the pathogenesis of RA. ' Some cross sectional studies have shown that raised levels of IC in serum and synovial fluid (SF) are associated with an active joint disease $\mathrm{e}^{2-4}$ or with the presence of extra-articular features (EAF), ${ }^{5-7}$ whereas other authors found only a weak association or none at all between IC and disease parameters. ${ }^{7-9}$ This discrepancy might be partially due to differences in the sensitivity of various assays for various types of IC. ${ }^{10}$ With the development of

Accepted for publication 8 April 1986

Correspondence to Dr M-L Westedt, Department of Rheumatology, Staff Centre C2Q, University Hospital, PO Box 9600. 2300 RC Leiden, The Netherlands.

*This work was presented at the British Society for Rheumatology, London, November 1985. specific methods for the detection of circulating IC containing not only $\operatorname{IgG}$ but also $\operatorname{IgA}$, IgM, and complement $(\mathrm{C} 3$ or $\mathrm{C} 4)$ it is now possible to investigate the occurrence and significance of various classes of circulating IC. . $^{11} 12$

In a recent cross sectional study we found a positive association between the levels of $\mathrm{Clq}$ binding activity (C1qBA), IC containing IgG and IgA (IgG IC, IgA IC) and both joint severity (i.e., the Ritchie index) and the presence of rheumatoid vasculitis (RV). ${ }^{13}$ Relatively little is known about the relevance of longitudinal measurements of circulating IC and rheumatoid factor (RF) as indicators of disease activity within individual RA patients. Some studies have shown that a decrease of IC levels and the amount of RF are associated with a reduction of joint inflammation ${ }^{14}$ or diminished activity of a systemic disease, e.g., $\mathrm{RV},{ }^{16}{ }^{17}$ when patients are treated with a remission inducing drug. Few prospective studies have been published concerning the value of RF and IC for the 
prediction of disease activity. ${ }^{18-20}$ Most of the methods used for the determination of IC in these studies were based on the detection of complement or IgG within the IC. Other components of IC have seldom been investigated.

The aim of the present study was to determine whether changes in disease activity correlated with fluctuations of the class specific IC and RF titres and whether any of these assays can predict joint disease activity or development of EAF. In addition, possible correlations were sought between measurements of circulating IC at the initial clinical assessment of patients with RA who present early and the development of erosions one year later.

\section{Patients and methods}

\section{PATIENTS}

Fifty seven of the original 60 seropositive patients with definite or classical $\mathrm{RA}^{21}$ described elsewhere ${ }^{13}$ were followed up for at least one year. Three patients were lost to follow up; one refused further evaluation, one died of septicaemia, and the other died with severe RV. The patients entered this prospective study over a period of 12 months, and after 30 months the study was closed. On entry thirty two patients had a disease duration of less than one year (early RA) and 25 patients had established RA with a disease duration of at least two years. Of the patients with established RA, seven had signs of RV at entry. Vasculitis was defined by the presence of one or more of the following features: deep ulcers, severe nailedge thrombi, digital gangrene, palpable purpura, or neuropathy. In six of seven patients this was histologically proved by biopsies of skin, muscle, or kidney at the initial assessment. One patient in the early RA group developed RV after one year of the study with skin eruptions. The involved skin had leucocytoclastic vasculitis. All clinical measurements were performed by the same physician (MLW) at six month intervals with special attention to the signs of EAF such as nodules, pleuritis, pericarditis, episcleritis, neuropathy, Felty's syndrome, or vasculitis. Joint disease activity was scored not only as joint tenderness according to the Ritchie articular index ${ }^{22}$ but also as the total number of swollen joints. Hand, wrist, and forefoot radiographs of the patients with early RA were taken at entry and one year later. At the end of the study the radiographs of each patient were assessed blindly by one of us (AC) according to the Atlas of Standard Radiographics. ${ }^{23}$ Erosions were scored according to a five point scale: no abnormalities $(0)$, dubious (1), mild but definite (2), moderate erosions (3), and severe destructive lesions or ankylosis, or both (4).
The total number of joints in the hands, wrists, and feet with radiographic scores of grade 2 or more was recorded (maximum of 42 joints for each patient).

\section{LA B ORATORY ASSESSMENT}

At the time of physical examination (mid-morning) blood was collected for laboratory analysis. Serum was stored at $-70^{\circ} \mathrm{C}$ before testing. RF were detected by the Waaler-Rose agglutination test ${ }^{24}$ and the latex fixation test. ${ }^{25}$ IC were measured by the ${ }^{125} \mathrm{I}-\mathrm{Clq}$ binding assay $(\mathrm{C} 1 \mathrm{qBA})^{26}{ }^{27}$ and radioimmune polyethylene glycol 6000 (PEG) precipitation assays (for IC containing IgM, IgG, IgA, $\mathrm{C} 3$, and C4), ${ }^{11} 1228$ using 31/2\% PEG.

STATISTICAL ANALYSIS

Spearman's rank correlation coefficient was used to detect associations between laboratory values and clinical parameters for disease activity at the time of examination and also six months later. Patients with RA were divided into two groups, one showing EAF at more than $50 \%$ of all clinical evaluations and the other with EAF at less than $50 \%$ of the hospital visits. The differences in RF titres of IC levels between these two groups were assessed by the Wilcoxon two sample test (see Table 2). Possible relationships between the results of IC assays and RF tests and increases of the number of radio graphic erosions were assessed by the Spearman's rank correlation coefficient for each patient separately. The associations between the individual parameters were assessed with Student's $t$ test to examine the hypothesis that the (weighted) mean of the correlation coefficient was equal to zero. ${ }^{19}$ The relation within patients between fluctuations in the occurrence of EAF and RF titres or IC levels was studied by computing for each patient the mean test value during the periods when the patient did and did not show EAF. The sign test was then used to establish whether the difference between these two mean levels was significantly more often positive than negative.

\section{Results}

JOINT DISEASE ACTIVITY, SERUM IC

LEVELS, AND RF TITRES

Correlation analysis between the levels of IC and RF titres and clinical parameters at successive times of assessment (Table 1) showed that IC measured by the C1qBA and IgM IC had the most significant correlations. In most instances, however, a significant correlation was also observed between disease activity and the other laboratory parameters. At the initial assessment RF titres did not correlate with either the number of swollen joints or the Ritchie 
Table 1 Correlation between indices of joint activity and laboratory parameters in the present study

\begin{tabular}{|c|c|c|c|c|c|c|c|}
\hline \multirow{2}{*}{$\begin{array}{l}\text { Time of assessment } \\
\text { after entry (months) }\end{array}$} & & \multicolumn{6}{|c|}{ Correlation coefficient } \\
\hline & & Latex & $\begin{array}{l}\text { Waaler- } \\
\text { Rose }\end{array}$ & $C I q B A$ & $\operatorname{Ig} G I C$ & $\operatorname{IgA} I C$ & $\operatorname{Ig} M I C$ \\
\hline \multirow[t]{2}{*}{$0(n=57) \dagger$} & Swollen joints & $0 \cdot 18$ & $0 \cdot 15$ & $0 \cdot 45^{* * *}$ & $0 \cdot 39^{* *}$ & $0 \cdot 29^{*}$ & $0.41^{* *}$ \\
\hline & Articular index & $0 \cdot 11$ & $0 \cdot 19$ & $0 \cdot 44^{* *}$ & $0.42^{* *}$ & $0 \cdot 35^{* *}$ & $0 \cdot 52^{* * *}$ \\
\hline \multirow[t]{2}{*}{$6(n=57)$} & Swollen joints & $0 \cdot 43^{* *}$ & $0 \cdot 44^{* *}$ & $0 \cdot 47^{* * *}$ & $0.43^{* *}$ & 0.26 & $0 \cdot 14$ \\
\hline & Articular index & $0.42^{* *}$ & $0 \cdot 33^{*}$ & $0.37^{* *}$ & $0 \cdot 36^{* *}$ & $0 \cdot 31^{*}$ & $0 \cdot 43^{* *}$ \\
\hline \multirow[t]{2}{*}{$12(n=57)$} & Swollen joints & $0 \cdot 40^{* *}$ & $0.41^{* *}$ & $0 \cdot 42^{* *}$ & $0.42^{* *}$ & $0 \cdot 37^{* *}$ & $0 \cdot 38^{* *}$ \\
\hline & Articular index & $0.42^{* *}$ & $0 \cdot 38^{*}$ & $0 \cdot 42^{* *}$ & $0.44^{* *}$ & $0 \cdot 40^{* *}$ & $0 \cdot 39^{* *}$ \\
\hline \multirow[t]{2}{*}{$18(n=36)$} & Swollen joints & $0 \cdot 40^{* *}$ & $0 \cdot 23$ & $0 \cdot 29^{*}$ & $0 \cdot 34^{*}$ & $0.46^{* *}$ & $0 \cdot 39^{* *}$ \\
\hline & Articular index & $0.41^{* *}$ & $0 \cdot 28$ & $0 \cdot 36^{*}$ & $0 \cdot 27$ & $0 \cdot 32^{*}$ & $0 \cdot 56^{* * *}$ \\
\hline \multirow[t]{2}{*}{$24(n=36)$} & Swollen joints & $0 \cdot 48^{* *}$ & $0.53^{* *}$ & $0 \cdot 40^{*}$ & $0 \cdot 31$ & 0.29 & $0 \cdot 42^{*}$ \\
\hline & Articular index & $0.41^{*}$ & $0 \cdot 35$ & $0 \cdot 21$ & $0 \cdot 28$ & $0 \cdot 36$ & $0 \cdot 28$ \\
\hline
\end{tabular}

${ }^{*} \mathrm{p}<0.05 ;{ }^{* *} \mathrm{p}<0.01 ;{ }^{* * *} \mathrm{p}<0.001$.

$+n=$ number of patients.

index for the total group of 57 patients, whereas a significant correlation was found during the follow up period. This difference occurred because of the lack of association between RF titres and joint activity parameters (number of swollen joints or articular index) of the 32 patients with early disease. In contrast, IC levels were similar in patients with longstanding RA and in those with early disease (data not shown). No association was found between the presence of C3 IC or C4 IC and the activity parameters.

ASSOCIATION BETWEEN MEAN SERUM IC LEVELS AND RF TITRES AND THE INCIDENCE OF EAF

There was an association between the occurrence of EAF and the mean level of IC and titre of RF. Nine patients who showed no EAF had a mean C1qBA level of $31 \mu \mathrm{mol} / \mathrm{ml}, 18$ patients who had signs of EAF at half or less of all their physical examinations had a mean level of $42 \mu \mathrm{mol} / \mathrm{ml}, 13$ patients who had

Table 2 Association between raised mean values of circulating IC or $R F$ and an increased incidence of EAF $(<50 \%)$ during the follow up study $(n=57)$

\begin{tabular}{ll}
\hline Test & $p$ Value $^{*}$ \\
\hline Latex & NS \\
Waaler-Rose & NS \\
ClqBA & $<0.001$ \\
IgG IC & $<0.05$ \\
IgA IC & $<0.005$ \\
IgM IC & $<0.005$ \\
C4 IC & $=0.05$ \\
C3 IC & NS \\
\hline
\end{tabular}

${ }^{*} \mathrm{p}$ Values were calculated with Wilcoxon's two sample test.
EAF at more than $50 \%$ of all examinations had a mean IC level of $84 \mu \mathrm{mol} / \mathrm{ml}$, and 17 patients who had signs of EAF throughout the study had a mean IC level of $203 \mu \mathrm{mol} / \mathrm{ml}$. Similar differences in mean levels of IC containing IgG, IgA, and IgM were found for these groups of patients (data not shown).

As shown in Table 2 raised levels of IC containing ClqBA, IgG, IgA, and IgM were associated with EAF. When patients with vasculitis were excluded, however, the association held only for C1qBA IC and IgM IC.

\section{RELATION OF RF TITRES AND IC LEVELS TO DISEASE ACTIVITY AND THE OCCURRENCE OF EAF}

To investigate the possible relation between clinical and laboratory parameters within individual patients in a longitudinal study correlation coefficients were computed for each patient. The analysis was based on between three and six observations for each patient. To compensate for the unequal numbers of observations taken into account these correlation coefficients were normalised by multiplying them by $\sqrt{(n-1)}$ (i.e., the approximate standard error), $n$ being the number of observations of the patients. Student's one sample $t$ test was then used to examine whether the mean correlation coefficient differed significantly from zero. ${ }^{19}$ Table 3 shows the results of these analyses. The fluctuation of RF titre, C1qBA IC, and IgM IC correlated significantly with the disease activity (number of swollen joints or articular index, or both) IgG IC correlated significantly only with the articular index, and IgA IC did not correlate with either parameter of joint disease activity.

Only 30 patients showed fluctuations of EAF during this longitudinal study. Nine patients showed 
Table 3 Correlation of RF and IC levels with joint disease activity during the course of the disease in individual patients $\dagger$

\begin{tabular}{llll}
\hline Assays & $\begin{array}{l}\text { No of } \\
\text { patients } \ddagger\end{array}$ & $\begin{array}{l}\text { Mean }(S D) \text { of normalised } \\
\text { Spearman's rank correlation } \\
\text { coefficients }\left(r_{s} \sqrt{n-1}\right) \text { for: }\end{array}$ \\
\cline { 2 - 4 } & & Swollen joints & Articular index \\
\hline Latex & 50 & $0.65(0.93)^{* * *}$ & $0.43(0.98)^{* *}$ \\
Waaler-Rose & 55 & $0.46(1.08)^{* *}$ & $0.51(1.05)^{* * *}$ \\
C1qBA & 54 & $0 \cdot 51(0.87)^{* * *}$ & $0.76(0.92)^{* * *}$ \\
IgG IC & 54 & $0 \cdot 28(1.13)$ & $0.39(1.02)^{*}$ \\
IgA IC & 44 & $0.32(1.06)$ & $0.20(1.09)$ \\
IgM IC & 57 & $0 \cdot 47(0.94)^{* * *}$ & $0.39(0.98)^{* *}$ \\
\hline
\end{tabular}

${ }^{*} \mathrm{p}<0.01 ;{ }^{* *} \mathrm{p}<0.005 ;{ }^{* * *} \mathrm{p}<0.001$.

†Significance of the deviation of the mean correlation coefficient (r) from zero assessed by Student's one sample $t$ test.

$\ddagger$ Correlation coefficients could not be calculated for all patients

no signs of EAF during the entire study period, and 18 patients always had EAF when examined. In the group of 30 patients differences between the RF titre or IC level during the stage when EAF were present or absent were computed (Table 4). Application of the sign test showed that the levels of IgG IC and IgA IC in each patient were significantly higher in the presence of EAF. Complement C3 IC and $\mathrm{C} 4 \mathrm{IC}$ were found infrequently and were not related to joint disease parameters during this longitudinal study. They were always present in sera of three patients with recurrent active vasculitis. In four other patients the C3 IC and C4 IC became undetectable at the same time as signs of vasculitis disappeared after treatment with corticosteroids or cytostatics, or both.

VALUE OF IC AND RF FOR PREDICTION OF JOINT ACTIVITY AND OCCURRENCE OF EAF When RF titres or IC levels of individual patients were compared with disease activity parameters

Table 4 Association between fluctuation in the occurrence of EAF and laboratory parameters in individual patients in the follow up study ( $p$ values calculated with the sign test)

\begin{tabular}{lll}
\hline Assays & No of patients & Significance $(p)$ \\
\hline Latex & 30 & NS \\
Waaler-Rose & 30 & NS \\
C1qBA & 29 & NS \\
IgG IC & 30 & $<0 \cdot 01$ \\
IgA IC & 29 & $<0 \cdot 05$ \\
IgM IC & 29 & NS \\
C4 IC & 29 & NS \\
C3 IC & 29 & NS \\
\hline
\end{tabular}

measured six months later significant correlation coefficients were not found (data not shown). Thus $\frac{}{\infty}$ these tests were in no way predictive of joint disease activity. Furthermore, RF titres and IC levels did not predict the development of EAF.

VALUE OF CIRCULATING RFANDIC FOR THE PREDICTION OF AN INCREASE IN JOINT EROSIONS

To investigate the relation between laboratory parameters and the development of radiological abnormalities radiographs were taken of each patient with early disease at entry to the study $(n=32)$ and again after approximately one year. Of the various RF and IC tests, only the level of IgA IC at the initial assessment was predictive of an increase of radiological lesions after one year $(r=0.72$; $\mathrm{p}<0.001)$. Furthermore, a significant association $(p<0.001)$ was found between the increase of the total number of joints with erosions (grade 2 or $\frac{\text { ㄱ }}{3}$ more) in each patient and the presence of $\operatorname{IgA}$ IC (Fig. 1). All six patients who had raised IgA IC levels showed a significant increase in the number of joints with erosions. This suggests that this test $\stackrel{\mathbb{Q}}{-}$ could serve as a specific indicator for the develop $\overrightarrow{0}$ ment of erosive arthritis in early RA.

\section{Discussion}

In an early cross sectional study we found that patients with active joint disease, as measured by the Ritchie articular index, have raised circulating IC levels and higher mean levels of IC more frequently than patients with inactive disease. ${ }^{1.3}$

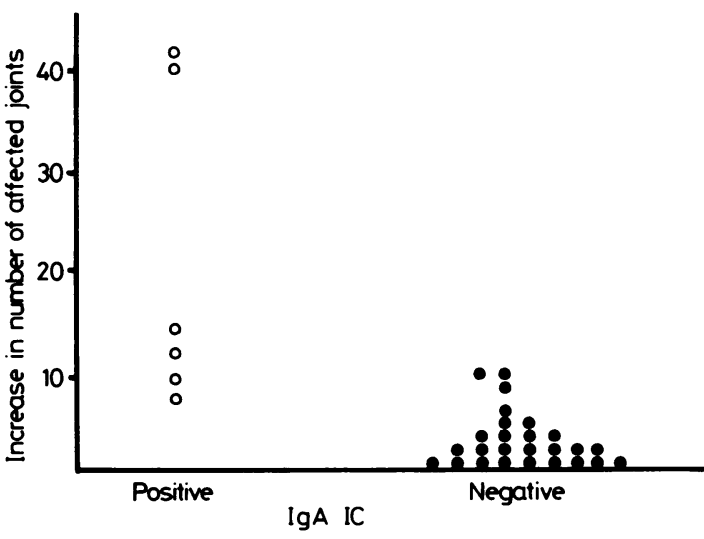

Fig. 1 Increase in the number of joints with radiological erosive disease after one year of observation of patients with $(O)$ or without $(O)$ circulating $\operatorname{Ig} A$ IC at the start of the study. $(p<0 \cdot 001$, Wilcoxon's rank sum two sample test). 
Circulating IC were also associated with the presence of EAF, including RV. The results of the present longitudinal study made at initial assessment, and also at almost all subsequent assessments, confirm the earlier findings.

The absolute values of the IC and RF were found to correlate significantly with disease activity as assessed by either the Ritchie index or the number of swollen joints (Table 1), though the correlation coefficients were not high. Similar correlations were found between high mean levels of IC and the presence of EAF during our study (Table 2). When signs of clinical RV were excluded from this patient group, however, only IC containing $\mathrm{C} 1 \mathrm{qBA}$ and IgM correlated significantly with EAF. This is explained by the fact that IgA IC, IgG IC, C3 IC, and C4 IC were associated predominantly with RV. IgM IC alone may not cause RV, since in one study signs of vessel inflammation were absent in biopsy specimens of clinically uninvolved skin even though IgM deposits were present in the vessel wall. ${ }^{29}$ IC levels determined by the C1qBA test correlated significantly with the presence of IgM deposits in the skin. ${ }^{30}$

An overall correlation between the level of circulating IC and various measurements of disease activity (joint activity, extra-articular features, or erythrocyte sedimentation rate (ESR)) had been found in a large number of clinical studies, though published values differ considerably. ${ }^{2-7}$ This relationship has been substantiated by one of the most extensive cross sectional studies. ${ }^{31}$ According to these authors none of the tests used to detect IC offered diagnostic advantages over other established laboratory tests. This report raised several questions. Are IC determinations clinically useful for the monitoring of disease activity in individual patients? Can they predict the course of inflammatory parameters of joint disease, the occurrence of EAF, or the development of erosions?

The data from the present longitudinal study indicate that RF and some IC tests (C1qBA and IgM IC) correlate significantly with clinical indices for joint activity (Table 3). Of all these tests, we found the ClqBA to be associated most significantly with fluctuations in Ritchie articular index, and this laboratory parameter might be useful for monitoring rheumatoid joint disease. Our data indicate, however, that the RF and IC tests have no value as prognostic indicators of disease activity (joint activity or EAF). Only a few reports are available concerning serial studies on RF and circulating IC in relation to articular manifestations. ${ }^{14-19} 3233$ In general, our data confirm the results reported by these authors, though most of the studies reported previously were based on a small number of obser- vations in each patient, or the period covered was relatively short. Our results concerning the lack of predictive value of IC and RF for joint disease activity during the course of the disease are compatible with recent reports, ${ }^{20} 33$ which suggest that the circulating IC are epiphenomena and not pathogenic. If so, the IC and RF would not be more clinically specific for joint destruction than, for example, the ESR.

The occurrence of IgA IC in RA, and particularly in patients with EAF, is of interest. ${ }^{13}$ In our patients only changes in IgA IC and IgG IC paralleled changes in EAF (Table 4), and IC did not predict the occurrence of EAF. IC containing $\mathrm{C} 3$ and $\mathrm{C} 4$ were found so infrequently that statistical tests were not applicable, but it was obvious that they occurred only in patients with recurrent vasculitis. In two patients only slightly increased IC levels were detected at the first clinical evaluation, but a few weeks later active vasculitis developed, and the IC levels were strongly raised, showing the lack of predictive value in circulating IC measurement.

Few studies have been published on serial determinations of circulating IC in patients with RV treated with remission inducing drugs. ${ }^{16}{ }^{17}$ In these studies concentrations of IC and RF decreased with the disappearance of vasculitis, and one author suggested that IC containing IgG RF might be responsible for the development of vasculitis. ${ }^{17}$ Special tests to assess class specific immunoglobulins containing IC were performed in only one study, and in agreement with our observations these investigators found an association between the occurrence of EAF and the demonstration of IgA IC in RA patients. ${ }^{34}$

Although levels of IgA IC did not significantly coincide with changes in joint disease activity in our study, they did correlate significantly with the appearance of EAF. The established associations between IgA IC and EAF, ${ }^{13}{ }^{34}{ }^{35}$ between EAF and the development of erosive disease, ${ }^{36}{ }^{37}$ and between IgA IC and marked erosive changes in early $\mathrm{RA}$, found in the present study, suggest that IgA IC may be a good marker of the development of erosive disease. It has been suggested that IgA RF is less easily cleared from the joints than IgG RF and IgM RF and can spill over into the peripheral circulation. ${ }^{19}$ Whether the same applies to IgA IC is uncertain, but venous blood from limbs with actively inflamed joints has a higher content of IgA IC than arterial blood from the same limb. ${ }^{38}$ Involvement of $\operatorname{IgA}$ in the pathogenesis of the rheumatoid disease seems likely, especially since serum IgA levels are frequently raised in RA patients and a fall in the $\operatorname{IgA}$ level coincided with improvement of RA in patients treated with disease modifying drugs. ${ }^{39}$ Moreover. 
the presence of IgA IC in early RA appears to be associated with an unfavourable course of RA, as already found for $\operatorname{IgA} R$ R. $^{18} 19$

In summary, determination of $\mathrm{RF}$ and class specific IC did not yield more information about the clinical state or the prognosis of disease activity in RA patients than physical examination or other common laboratory tests. The same conclusion was drawn from a follow up study in patients with SLE. ${ }^{40}$ It is known that not all IC are functionally similar, however, and studies on IC combined with functional tests to assess phagocytosis and clearance of IC might yield more information about the role of IC in the pathogenesis of the often erratic course of RA and contribute to the understanding of this disease. ${ }^{41}$ Because IgA IC may precede the development of erosive arthritis further studies on the presence of these IC in early arthritis are warranted.

This study was supported by the Dutch League against Rheumatism. The authors wish to thank Miss J Schouten and Mrs $\mathrm{H} \mathrm{H}$ de Rooij-Dijk for their technical assistance, Mrs J Hins-Benjamins for the preparation of the manuscript, and $\mathrm{Dr} E$ de Vries for constructive criticism. Mrs I Seeger-Wolf corrected the English text.

\section{References}

1 Zvaifler $\mathrm{N} \mathrm{J}$. The immunopathology of joint inflammation in rheumatoid arthritis. Adv Immunol 1973; 16: 265-336.

2 Nydegger U E, Zubler R H, Gabay R, et al. Circulating complement breakdown products in patients with rheumatoid arthritis. Correlation between plasma C3d, circulating immune complexes, and clinical activity. J Clin Invest 1977; 59: 862-8.

3 Halla J T, Volanakis J E. Schrohenloher R E. Immune complexes in rheumatoid arthritis sera and synovial fluids. A comparison of three methods. Arthritis Rheum 1979; 22: 440-8.

4 Roberts-Thomson P J, Neoh S H, Bradley J, Milazzo S C. Circulating and intra-articular immune complexes in rheumatoid arthritis: a comparative study of the $\mathrm{Clq}$ binding and monoclonal rheumatoid factor assays. Ann Rheum Dis 1980; 39: 438-44.

5 Zubler R H, Nydegger U, Perrin L H, et al. Circulating and intra-articular immune complexes in patients with rheumatoid arthritis. Correlation of ${ }^{125} \mathrm{I}-\mathrm{Clq}$ binding activity with clinical and biological features of the disease. J Clin Invest 1976; 57: 1308-19.

6 Bourke B E, Moss I K, Mumford P. Horsfall A, Maini R N. The complement fixing ability of putative circulating immune complexes in rheumatoid arthritis and its relationship to extraarticular disease. Clin Exp Immunol 1982; 48: 726-32.

7 Gupta R C, McDuffie F C, Huston K A, et al. Comparison of three immunoassays for immune complexes in rheumatoid arthritis. Arthritis Rheum 1979; 22: 433-9.

8 Hay F C, Nineham L J, Perumal R, Roitt I M. Intra-articular and circulating immune complexes and antiglobulins (IgG and IgM) in rheumatoid arthritis: correlation with clinical features. Ann Rheum Dis 1979; 38: 1-7.

9 Sølling J, Sølling K, Lassen L. An evaluation of two methods for detection of circulating immune complexes in patients with rheumatoid arthritis. Scand J Rheumatol 1980; 9: 118-22.

10 Lambert P H, Dixon F J, Zubler R H, et al. WHO collaborative study for the evaluation of eighteen methods for detecting immune complexes in serum. J Clin Lab Immunol 1978; 1: $1-15$.
11 Valentijn R M, van Es L A, Daha M R. The specific detection of IgG, IgA and the complement components $\mathrm{C} 3$ and $\mathrm{C} 4$ in circulating immune complexes. J Clin Lab Immunol 1984; 14: 81-6.

12 Baldwin W M, van Es A, Valentijn R M, van Gemert G W, Daha M R. van Es $\mathrm{L} A$. Increased IgM and IgM complex-like material in the circulation of renal transplant recipients with primary cytomegalovirus infection. Clin Exp Immunol 1982; 50: $515-25$.

13 Westedt M-L, Daha M R, de Vries E, Valentijn R M, Cats A. IgA containing immune complexes in rheumatoid vasculitis and in active rheumatoid disease. $J$ Rheumatol $1985 ; 12: 449-55$. क

14 Nineham L J, Hay F C, Male D K, Roitt I M, Young A, $\overrightarrow{0}$ Perumal R. Immune complexes in rheumatoid arthritis: correlations with clinical features and effects of gold. Protides of Biol $\vec{\omega}$ Fluids 1979; 26: 179-82.

15 Lessard J, Nunnery E, Cecere F, McDuffy S, Pope R M. فे Relationship between the articular manifestations of rheuma- $\overline{2}$ toid arthritis and circulating immune complexes detected by $\overrightarrow{0}$ three methods and specific classes of rheumatoid factors. $J$ Rheumatol 1983; 10: 411-7.

16 Abel Th, Andrews B S, Cunningham P H, Brunner C M, Davis $\infty$ J S, Horwitz D A. Rheumatoid vasculitis: effect of cyclophosphamide on the clinical course and levels of circulating immune complexes. Ann Intern Med 1980; 93: 407-13.

17 Scott D G I, Bacon P A, Allen C, Elson C J, Wallington T. IgG rheumatoid factor, complement and immune complexes in $\bigcirc$ rheumatoid synovitis and vasculitis: comparative and serial studies during cytotoxic therapy. Clin Exp Immunol 1981; 43: $54-63$.

18 Teítsson I, Withrington R H, Seifert M H, Valdimarsson $\mathrm{H}$. Prospective study of early rheumatoid arthritis. I. Prognose value of IgA rheumatoid factor. Ann Rheum Dis 1984; 673-8.

19 Withrington R H, Teitsson I, Valdimarsson $H$, Seifert $M$ S Prospective study of early rheumatoid arthritis. II. Associatiø் of rheumatoid factor isotypes with fluctuations in disease activity. Ann Rheum Dis 1984; 43: 679-85.

20 Reeback J S, Silman A J, Holborow E J, Maini R N, Hay F C. 응 Circulating immune complexes and rheumatoid arthritis: a $\varrho$ comparison of different assay methods and early predictive $\overrightarrow{\vec{B}}$ value for disease activity and outcome. Ann Rheum Dis 1985; 44: 79-92.

21 Ropes M W, Bennet G A, Cobb S, Jacox R, Jessar R A. 1958 revision of diagnostic criteria for rheumatoid arthritis. Bull? Rheum Dis 1958; 9: 175-6.

22 Ritchie D M, Boyle J A, McInnes J M, et al. Clinical studies with an articular index for the assessment of joint tenderness in $\overline{0}$ patients with rheumatoid arthritis. $Q J$ Med 1968; 37: 393-8.

23 Atlas of standard radiographics of arthritis. In: Kellgren $\mathbf{J} \mathbf{H}$, ed. The epidemiology of chronic rheumatism. Vol 2. Oxford: O Blackwell Scientific, 1963.

24 Valkenburg H A. Human erythrocyte agglutination test $\mathrm{O}$ (HEAT). In: Kellgren J H, Jeffrey M R, Ball J, eds. The epidemiology of chronic rheumatism. Vol 1. Oxford: Blackwell Scientific, 1963: 330-3.

25 Klein F, Bronsveld W, Norde W, van Romunde L K J, Singer J M. A modified latex-fixation test for the detection of rheuma- $\sigma$ toid factors. J Clin Pathol 1979; 32: 90-2.

26 Zubler R H, Lange G, Lambert P H. Detection of immune complexes in unheated sera by a modified ${ }^{125}$ I-C1q-binding test. $\omega$ J Immunol 1976; 116: 232-5.

27 Kauffman R H, van Es L A, Daha M R. Aggregated human immunoglobulin $G$ stabilized by albumin: a standard for immune complex detection. J Immunol Methods 1979; 31: 11-2. \&

28 Valentijn $R$ M, van Es L A, Westedt M-L, Daha M R. The ${ }^{+}$ detection of circulating immune complexes containing im- $\square$ munoglobulin G. J Clin Lab Immunol 1984; 14: 73-9.

29 Westedt M-L, Meijer C J L M, Vermeer B J, Cats A, de Vries $\vec{D}$ E. Rheumatoid arthritis - the clinical significance of histo- and $\frac{\mathcal{P}}{\mathscr{Q}}$ 
immunopathogical abnormalities in normal skin. $J$ Rheumatol 1984: 11: $448-53$.

30) Westedt M L. Vermeer B J. Meijer C J L M. Daha M R. Cats A. Immuno- and histopathology of the skin in patients with rheumatoid arthritis. In: McDonald. ed. Immunodermatology. London: Butterworth. 1984: 225-9.

31 McDougal J S. Hubbard M. McDuffie F C. et al. Comparison of five assays for immune complexes in rheumatoid arthritis. Arthritis Rheum 1982: 25: 1156-66.

32 Berglund K. Laurell A-B. Nived O. Sjoholm A G. Sturfelt G. Complement activation. circulating $\mathrm{Clq}$ binding substances and inflammatory activity in rheumatoid arthritis: relations and changes on suppression of inflammation. Clin Lab Immunol 1980): 4: 7-14.

33 Highton J. Panayi G S. Shepherd P. Faith A. Griffin J. Gibson T. Fall in immune complex levels during gold treatment of rheumatoid arthritis. Ann Rheum Dis 1981: 40: 575-9.

34 Jans H. Halberg P. Lorenzen I. Circulating immune complexes in rheumatoid arthritis with extra-articular manifestations. Scand J Rheumatol 1983: 12: 215-8.

35 Melsom R D. Smith P R. Maini R N. Analysis of circulating immune complexes isolated by affinity chromatography from the sera of rheumatoid arthritis patients. Ann Rheum Dis 1984: 43: $116-7$.
36 Wawrzynska-Pagowksa A. Brzezinska B, Brzozowska M, et al. Observations on the symptoms and signs of 'early' rheumatoid arthritis in a prospective study. Acta Rheumatol Scand 1970; 16: 99-105.

37 Gordon D A. Stein J L. Broder I. The extra-articular features of rheumatoid arthritis: a systematic analysis of 127 cases. $A$ m J Med 1973; 54: 445-52.

38 Steven M M, Westedt M-L, Daha M R, de Vries E, Cats A. Comparison of immune complexes and complement components in arterial and venous blood of patients with rheumatoid arthritis. J Rheumatol 1986; 13: 71-8.

39 Delamere J P. Grindulis K. Farr M. Effects on rheumatoid activity of drug-induced changes in serum immunoglobulins, particularly selective IgA deficiency. Ann Rheum Dis 1983; 42: 231.

40 Valentijn $R$ M, van Overhagen $H$, Hazevoet $\mathrm{H} \mathrm{M}$, et al. Value of complement and immune complex determinations for patients with systemic lupus erythematosus to monitor disease activity. Arthritis Rheum 1985; 28: 904-13.

41 Daha M R, Westedt M L, Bos B, Krol H C, van Es L A, Cats A. Diminished uptake and degradation of soluble complexes of IgG (AIgG) by monocytes of patients with rheumatoid arthritis (RA) and vasculitis. Br J Rheumatol 1986; 25: 128. 